What are the next steps? First, more research should be aimed at increasing the effective usage of roads and streets and at diverting potential demand. Secendly, the available means of traffic restraint, and the manner in which they should be used, need detailed investigation. Thirdly, further investigation is needed not only of new road layouts but also of methods for determining how far the expenditure of large sums on Buchanan-style schemes can be justified. These are some of the big questions which the Buchanan Group has left unanswered but which, in the light of the Report, have become even more pressing than before. For guidance on some of these questions, if not for a complete answer, one may turn to Mr. Foster and to other economists at present working in this field.

\section{STOCHASTIC THEORIES OF TRAFFIC FLOW}

Mathematical Theories of Traffic Flow

By Frank A. Haight. (Mathematies in Science and Engineoring : a Series of Monographs and Textbooks, Vol. 7.) Pp. xi + 242. (New York: Academic Press, Inc.; London: Academic Press, Ino. (London), Ltd., 1963.) 728.

MATEMATICAL Theories of Traffic Flow is the first book to attempt a systematic treatment of vehioular traffic flow on individual roads or individual junctions from a mathematical (or rather a stochastic) point of view. It is highly mathematical and makes no concessions to those who desire guidance towards solving the practical problems of traffic engineering, so that in this respect its title is accurate.

Chapter 1 is an introduction to those parts of statistical theory which are needed. Chapter 2 is a similar introduction to queue theory. The material in these two chapters must be very well understood, together with the use of the Leplace transform, in order to follow the rest of the book. Chapter 3 examines the shapes of the curve relating vehicle flow (vehicles per unit time) to concentration (vehicles per unit distance) for a single lane suggested by statistical, car-following, fluid dynamic, and observational considerations. Their implications are explored. Chapter 4 considers the distribution in space and in time of vehicles on a single-track one-way road and gives the relatively simple formulæ connected with counting them. Chapter 5 considers the simple delay problem of pedestrians, or cars at a junction, waiting to cross a one-way road. (Cars queue, pedestrians do not.) The cases of free and of lightcontrolled junctions are both covered. The next two chapters are less systematic in treatment and explore more difficult problems. These include offside turners at junctions, multi-lane highways, sequences of traffic controls at junctions along a one-way road (3 pages), parking on a parking lot, merging into a lane of faster traffic (a most complicated problem), two-way traffic passing through a bottleneck and an extended treatment of fast cars overtaking slower vehicles in one direction on a twolane, two-way road. Each chapter occupies 25-35 pages.

The book is very well provided with references to sources and to related work: there are several hundred such references and nearly half are for 1960 or later. Thus it is clear that mathematical theories of traffic flow form a growing subject and, as treated in this book, this is mainly a recent one-which is not surprising, as traffic flow is here regarded as a source related to daily life of interesting and complicated applications of queueing theory. The Lighthill-Whitham theory of traffic flow on long, crowded roads is dismissed summarily in $2 \frac{1}{2}$ pages although it clearly comes within the title of the book. This is excused in the preface, where the author states:
"The present volume reflects my belief that the greatest development of traffic theory will take place in these directly connected areas [queueing theory, stochastic processes and mathematical probability], rather than by analogical variation of equations which apply to other substances".

Several of the sections end with an invocation to computing machinery for carrying the analysis further, but the author shows no interest in the methods by which a computer could do this. There is one mention of games theory (in relation to overtaking on a three-lane road), but no work in this direction is included: the book regards vehicles only as entities the behaviour of which is described by probability distributions and which are, usually, only influenced by the presence of neighbouring vehicles suffici. ently to avoid direct impacts. No malevolent or benevolent intelligence otherwise affects their motion.

The presentation of the material is informal and relies on intuitive ideas rather than being formal and axiomatic. References to earlier results in the book when they are being used are usually adequate, but sometimes the text implies that the step to the next equation is smaller than in fact it is. When this is combined with the very large number of errors in the printing of the formulæ and in the equation numbers referred to, it makes the book difficult to follow. There are frequent footnotes and comments which help to make the reasoning easier to follow, and it is clear that the author has been very thoughtful in this way in his manner of presentation: it is unfortunate that the printing errors remain, as these largely vitiate this effort. Each chapter ends with useful comments which relate its contents to other work. Readers should note that 'central moments' is used to mean moments about the origin and 'finite' to mean non-zero. The book does not include anything on the economics of transport, the purpose of transport, or the organization of transport over a twodimensional region.

The printing, page leyout, paper and binding are of a high standard and the contents list, author index and subject index are quite adequate. If it were not for the errors in the mathematios expressions and elsewhere it would be a good text-book on stochastic theories of traffic flow.

K. N. Chandler

\section{COSMICAL ELECTRODYNAMICS}

\section{Cosmical Electrodynamics}

Fundamental Principles. By Hannes Alfvén and Carl. Gunne Fälthammar. Second edition. (International Series of Monographs on Physics.) Pp. $x+228$. (Oxford: Clarendon Press; London: Oxford University Press, 1963.) 60s. net.

'HE first (1950) edition of Prof. Alfvén's Cosmical Electrodynamics had four chapters devoted to fundamental principles, and three concerned with applications to solar physics, geomagnetic storms, and cosmic rays. Since its appearance, the subjects which it treated have undergone a striking development. Accordingly, it is planned to replace it by a series of monographs. The first of these, the subject of the present review, is a modernized and extended version of the chapters earlier devoted to fundamental principles.

As before, the topics treated fall under three headings: the motion of charged particles, magnetohydrodynamics, and plasma theory. The list of new topics discussed is impressive. It includes, for example, the adiabatic invariants of the motion of a charged particle, the acceleration of particles to high energies, hydromagnetic waves in compressible fluids, magnetohydrostatio equilibrium and stability, the origin of cosmic magnetic fields, runaway electrons, frozen-in lines of force, and streams of mag. netized plasma. Prof. Alfvén's scientific work has been marked by his ability to use simple physical concepts to 